Canadian

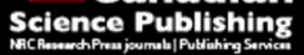

Botany

Botanique

\title{
The hooded mutant of Lathyrus odoratus (Fabaceae) is associated with a cycloidea gene mutation
}

\begin{tabular}{|r|l|}
\hline Journal: & Botany \\
\hline Manuscript ID & cjb-2017-0097.R1 \\
\hline Manuscript Type: & Article \\
\hline Date Submitted by the Author: & 11 -Oct-2017 \\
\hline $\begin{array}{r}\text { Complete List of Authors: } \\
\text { Is the invited manuscript for } \\
\text { consideration in a Special } \\
\text { Issue? : }\end{array}$ & $\begin{array}{l}\text { N/A } \\
\text { Cronk, Quentin; University of British Columbia, Botany }\end{array}$ \\
\hline $\begin{array}{r}\text { Keyword: } \\
\text { Rometal, identity gene, hooded mutant, CYCLOIDEA, Lathyrus odoratus, floral } \\
\text { homeotic mutation }\end{array}$ \\
\hline
\end{tabular}


ARTICLE

\section{The hooded mutant of Lathyrus odoratus (Fabaceae) is associated with a cycloidea gene mutation}

Christine Woollacott, and Quentin C.B. Cronk

Christine Woollacott and Quentin Cronk. Department of Botany, The University of British Columbia, 6270 University Blvd., Vancouver, BC V6T 1Z4, Canada.

Corresponding author: Quentin Cronk (email: quentin.cronk@ubc.ca).

Address: Department of Botany, The University of British Columbia, 6270 University Blvd., Vancouver, BC V6T 1Z4, Canada

Telephone: 1-604-781-6740 


\begin{abstract}
The hooded (hdd) floral mutant of Lathyrus odoratus (sweet pea) has a concave standard petal compared to the flat standard petal of the wild type. This trait was used by Bateson, Punnett and Saunders in early studies of Mendelian inheritance (c.1905). Here we provide four lines of evidence that this phenotype results from a mutation in the CYCLOIDEA2 (CYC2) gene. (1) CYC2 is expressed in the standard petals of wild-type L. odoratus, whereas the same methods fail to detect expression in $h d d$ plants. (2) Genomic sequencing reveals that the CYC2 gene sequence of $h d d$ plants is truncated at the TCP box and likely non-functional. (3) In a population of 118 plants, the $h d d$ phenotype cosegregated with the mutant allele of $C Y C 2$ without exception. (4) CYC2 is known to act as a dorsal petal identity gene. Consistent with this the standard petal in $h d d$ flowers has the epidermal and pigment characteristics of wing petals, indicating that the $h d d$ mutation results in a shift in dorsiventral petal-type identity. We conclude that the mutation in $C Y C 2$ is responsible for the hdd phenotype, and is therefore the L. odoratus equivalent of the lobed standard (lstl) mutant in Pisum.
\end{abstract}

Key words: floral homeotic mutation, Lathyrus odoratus, CYCLOIDEA, hooded mutant, petal identity gene 


\section{Introduction}

The sweet pea

Although Lathyrus odoratus L. is no longer a common model organism, it has a long history of being used in genetic research. It was one of the first organisms that was used to verify Mendel's work with the garden pea (Pisum sativum L.) in the early 1900s (Bateson 1902). The genetical phenomenon of linkage was first demonstrated in L. odoratus, although these observations were initially not correctly interpreted and did not directly lead to the development of modern chromosomal theory (Bateson et al. 1906; Carlson 2004). Because of its large flowers and the many pure-breeding horticultural inbred lines, L. odoratus is especially suited as a model organism with which to study flower development.

Lathyrus odoratus has papilionoid flowers with dorsiventral asymmetry. The style is twisted in L. odoratus, as in many other Lathyrus species, so bilateral symmetry is not perfect (Senn 1938). The flowers consist of three specialized petal types: one large standard (banner)

petal, two wing petals and two keel petals (Figure 1). The keel petals are often fused together and encase the carpel and stamens. Wild type L. odoratus is always a "bicolour" form in which the standard petal is a different colour (purple) from the wing and keel petals (blue) (Punnett 1923). A red and white bicolour flower similar to the ancient cultivar, 'Painted Lady,' is also said to occur in the wild (Bateson 1905).

Besides mutations in colour, some L. odoratus cultivars display mutations in the shape of their petals. One such floral type, called hooded ( $h d d)$, was examined in great detail by Punnett, Bateson and Saunders in the early 1900s (Bateson et al. 1906; Punnett 1923). Punnett (1919) 
called this locus 'E' (the 'Erect standard' locus of which hdd is the recessive form). Flowers of $h d d$ plants possess several shape and colour traits that distinguish them from wild-type flowers. The standard of $h d d$ plants is cupped and hood-shaped, rather than erect and banner-like (Figure 1). Strongly $h d d$ plants often possess two deep, symmetrical lobes on the edges of the standard petal, similar to the lobed standard 1 mutant of pea ( $P$. sativum), which is a cycloidea mutant (Wang et al. 2008). Although L. odoratus has a bicolour flower in wild-type, in the hdd mutant the standard is always the same colour as the wings. The $h d d$ standard petal also has a much less pronounced notch at the apex than in the wild-type. All hooded varieties apparently have the same genetic basis as they are all descended from the same original mutation (Punnett 1923).

\section{TCP-box genes influence plant development}

A class of genes known to affect petal form, and which are therefore candidates for the $h d d$ mutation, are the TCP-box genes. They are transcription factors that help regulate cell division and growth (Cubas et al. 1999; Zhong and Kellogg 2015; Fambrini and Pugliesi 2016). TCP-box genes are named from the genes TEOSINTE BRANCHED 1 (TB1) in Maize, CYCLOIDEA (CYC) in Antirrhinum, and PROLIFERATING CELL FACTOR 1 and 2 (PCF1, PCF2) in Oryza (Cubas et al. 1999). As a group, TCP-box genes share two evolutionarily conserved boxes which code for protein domains which likely function in DNA binding as well as in protein-protein interactions: the basic-Helix-Loop-Helix TCP structure and the arginine (R) rich 'R domain' (Cubas et al. 1999). TCP class genes are involved in many fundamental processes in plants being known to play roles in cell cycle control, plant architecture, lateral organ development and floral development (Luo et al. 1996; Cubas et al. 1999; Martín-Trillo and Cubas 2009; Xu et al. 2013). Expression of TCP genes is usually strongly regulated both 
spatially and temporally and thus they are able to influence growth and development at a fine scale (Cubas 2002).

\section{CYCLOIDEA-like genes control floral asymmetry in eudicots}

One subset of TCP genes (CYCLOIDEA (CYC)-like genes) are known to control floral asymmetry. This was first shown in Antirrhinum (Luo et al. 1996), in which CYC controls floral zygomorphy by specifying the within whorl radial organ identity of the dorsal corolla lobes (Luo et al. 1996; Hileman et al. 2003).

CYC-like genes have since been shown to play a major role in the control of floral zygomorphy in plants other than Antirrhinum. CYC-like TCP genes appear to be ubiquitous in eudicots, having been isolated in a great variety of plant species (Cubas et al. 1999; Broholm et al. 2008; Hileman 2014a; Hileman 2014b; Fambrini and Pugliesi 2017).

CYC-like genes are known from diverse legumes (Citerne et al. 2003). In Lotus japonicus, there are three genes orthologous to CYC and its close paralog DICHOTOMA (DICH) in Antirrhinum (Feng et al. 2006). However, specifically the $C Y C$ homolog $L j C Y C 2$ was found to control dorsal petal development and identity (Feng et al. 2006). Over-expression of $L j C Y C 2$ caused the papilionoid Lotus flowers to become increasingly radialized. In over-expression phenotypes there is less differentiation between the petals due to the wing and keel petals gaining a more standard-like form (Feng et al. 2006; Xu et al. 2016). This functional role of CYC2 in legumes is supported by mutant analysis. The squared standard mutant of Lotus is caused by a loss of function mutation in $L j C Y C 2$ and results in an oddly shaped standard that shares many wing petal characters (Feng et al. 2006; Xu et al. 2013; Xu et al. 2016). A CYC mutation in 
Pisum, called lobed standard 1 (lst1), has a similar phenotype to the squared standard mutant of Lotus

$C Y C$-like genes are therefore likely candidate genes underlying the $h d d$ mutant of Lathyrus odoratus for the following reasons:

(1) Genes of the Legume CYC2 clade (also called CYC1B) control dorsal (standard) petal identity in papilionoid flowers such as Lotus, Lupinus and Pisum (Citerne et al. 2006; Feng et al. 2006; Wang et al. 2008; Xu et al. 2013; Xu et al. 2016) and the hdd floral phenotype shows a visible change in the standard petal.

(2) The lack of a bicolour hdd flower indicates that the standard petal may have undergone a shift in dorsal petal identity and is no longer distinct from the wing petals. As legume CYC2 functions in establishing dorsal petal identity (Citerne et al. 2006; Cronk 2006; Feng et al. 2006) it is a likely candidate gene.

(3) The hdd mutation only affects the standard petal. Legume CYC2 expression is strictly limited to the standard petal during flower development in papilionoid legumes (Citerne et al. 2006; Feng et al. 2006; Ojeda et al. 2017).

(4) The hdd phenotype is similar in many aspects to the squared standard 1 (squl) and lobed standard 1 (lst 1 ) mutants in Lotus and Pisum which are caused by changes in CYC2 expression. These mutations, like $h d d$, influence the shape and curvature of the standard petal (Feng et al. 2006; Wang et al. 2008).

The aim of this paper is therefore to determine if the $h d d$ mutation is caused by a change affecting a member of the CYCLOIDEA gene family. As CYC in Antirrhinum and CYC2 in legumes are well known to be dorsal petal identity genes (e.g. Hileman, Kramer and Baum 2003; Cronk 2006) we also wished to see whether the hooded mutant involves identity shifts between 
petals, as has been noted in other legumes (e.g. Citerne, Pennington and Cronk 2006). Scanning electron microscopy (SEM) was therefore used to examine the epidermal surfaces of $h d d$ and wild-type L. odoratus, as epidermal characters are good markers of dorsiventral petal identity in legumes (Ojeda et al. 2009).

\section{Materials and Methods}

\section{Tissue sampling, DNA and RNA extraction}

For genomic DNA extraction leaf tissue samples were collected and stored briefly in silica gel before extraction. The dried tissue was pulverized using a drill apparatus that could be fitted with sterile acrylic grinding bits. Genomic DNA was extracted from the dried leaf tissue using the Plant DNAzol Reagent protocol provided by the manufacturer (Invitrogen, Carlsbad, USA). The concentration of the genomic DNA was analyzed using a Nanodrop Spectrophotometer. PCR checks using ITS primers were then conducted to ensure that all the DNA samples were capable of undergoing PCR amplification.

Lathyrus odoratus floral tissue for RNA extraction was collected from unopened buds approximately one to one-and-a-half centimetres in length when all floral organs had been formed, but pollen had not yet been released. L. odoratus buds were dissected and separated into standard, wing and keel petal types prior to immersion in liquid nitrogen. Tissue samples were stored at $-80^{\circ} \mathrm{C}$ to prevent RNA degradation. Two wild-type L. odoratus cultivars (i.e. inbred lines) were used ('Cupani', 'America') and three hooded cultivars ('Annie B Gilroy', 'Miss Ellen Willmott', 'Lady’s Bonnet'). These varieties are well characterized and commercially available (Rice 2002). They are 
inbred lines with no detectable phenotypic variation between individuals when grown under uniform conditions. For each variety, one pooled tissue sample containing 3-5 petals was taken for each petal type, harvested from multiple flowers and individuals.

RNA extractions used the Concert Plant RNA Reagent (Invitrogen, Carlsbad, USA), following manufacturer's protocol. Purification of the extracted RNA, by removing the residual DNA contamination, was performed using the TURBO DNA-free ${ }^{\mathrm{TM}}$ kit produced by Ambion (Carlsbad, USA). To test for genomic contamination in Lathyrus RNA extractions, PCR reactions using RNA with Histone 4 (H4) primers (SL1815/SL0363 of Wang et al. 2008) were conducted. In rare cases where residual genomic DNA was detected, an additional DNase treatment was performed. Quantification of RNA was performed using a Nanodrop Spectrophotometer. The purified RNA was then used to produce cDNA with oligo(dT) primers using the RevertAid H minus First Strand cDNA Synthesis Kit (Fermentas, Burlington, CA), following the manufacturer's instructions.

RT-PCR

RT-PCR reactions were carried out using floral cDNA and primers designed to amplify CYCLOIDEA sequences in the closely related species Pisum sativum. Primers specific to Pisum CYC2 (SL0868/SL0970) and CYC1 (SL0932/SL0933) published in Wang et al. (2008, suppl. mat.) were used to examine expression patterns of homologs in Lathyrus odoratus, as they cross-amplified perfectly in L. odoratus. SL0868/SL0970 amplify a portion of LoCYC2 corresponding to nucleotides 564 to 1278 . General Histone 4 (H4) primers SL1815/SL0363 were used as a control (Wang et al. 2008). PCR reactions were carried out in an Eppendorf Mastercycler Gradient thermocycler with the following conditions: $94^{\circ} \mathrm{C}$ for $3 \mathrm{~min}$ for initial denaturation, and then 34 cycles of 1 minute at $94^{\circ} \mathrm{C}$ for denaturation, 1 minute at $50^{\circ} \mathrm{C}$ for annealing, 90 seconds at $72^{\circ} \mathrm{C}$ for extension. For each variety, RT-PCR experiments were replicated between 3 and 5 times, as a check for consistency. 
Isolating CYCLOIDEA genomic sequence from wild-type and hooded Lathyrus odoratus

Partial sequence of $\mathrm{LoCYC} 2$ was obtained through direct sequencing and cloning using published degenerate LEGCYC/F1 and LEGCYC/R1 primers, based on highly conserved regions of the gene, as previously described (Citerne et al. 2003). Extensive cloning indicated that LoCYC2 is single copy in L. odoratus. This is to be expected as L. odoratus is diploid and CYC2 is single copy in the closely realted Pisum sativum. All PCR reactions were carried out in an Eppendorf Mastercycler Gradient thermocycler using the conditions outlined in Citerne et al. 2003. In order to extract individual sequences from the mixture of amplified DNA fragments we used a TOPO® TA Cloning Kit for Sequencing (Invitrogen, Carlsbad, USA) following the protocol provided by the manufacturer. Partial sequence was also amplified using primers from Wang et al. (2008):

SL0868/SL0970, LjSSR1084/LjSSR1085, and SL0773/SL0970 (for LoCYC2) and SL0932/SL0933 (for $L o C Y C 1$ ).

Reactions were carried out in an Eppendorf Mastercycler Gradient thermocycler at the following conditions: $94^{\circ} \mathrm{C}$ for 3 min for initial denaturation, and then 34 cycles of 1 minute at $94^{\circ} \mathrm{C}$ for denaturation, 1 minute at $55^{\circ} \mathrm{C}$ for annealing, 90 seconds at $72^{\circ} \mathrm{C}$ for extension.

With partial sequence from $\mathrm{LoCYC2}$, it was possible to extend the length of known sequence using the GenomeWalker Universal Kit (Clontech, Mountain View, CA). This kit requires the construction of a DNA library as well as nested PCR and cloning to isolate the successfully amplified gene fragments. Using a DNA library constructed from wild-type ('Cupani') DNA, the full LoCYC2 homolog was sequenced as well as approximately 300 base pairs of the promoter region in addition to over 1000 base pairs of $L o C Y C 2$ sequence from the $h d d$ cultivar 'Annie B Gilroy' chosen as it is an original parent of the F2 population (see below). The full length wild-type $L o C Y C 2$ open reading frame (ORF) sequence is deposited in GenBank (accession number: MG281955) along with the sequence of the $h d d$ mutant allele (accession number: MG281956). 


\section{Crossing}

A wild type variety 'Cupani' and the hooded 'Annie B Gilroy' were selected as parents. In order to check against accidental selfing despite careful emasculation, the recessive $h d d$ variety was chosen as the maternal plant. No selfing was observed in the F1. The F1 plants were grown and allowed to naturally self-pollinate to produce the F2 generation. Under cultivation the species is entirely self-pollinating as honey-bees and bumble bees are not strong enough to work the specialized pollination mechanism (Westerkamp 1993). Nevertheless, to remove the possibility of accidental cross-pollination seed production was conducted in a greenhouse kept closed during pollination in order to exclude insects during the pollination period.

\section{Plant growth}

Seeds were planted in vermiculite and placed in a greenhouse seed germination bed at $20^{\circ} \mathrm{C}$ for germination. Following germination, the seedlings were transplanted into $15 \mathrm{~cm}$ diameter pots filled with sterile soil-free potting compost. Approximately half of the F2 population was planted in an outside plot at the University of British Columbia. The other half of the F2 population was retained under greenhouse conditions at a mean temperature of $20^{\circ} \mathrm{C}$. Greenhouse plants were watered daily, whereas those outside were watered every three to five days.

\section{Determination of alleles in F2}

In order to determine if the $h d d$ floral phenotype is inherited with the mutant allele of LoCYC2, eighteen wild-type-specific and eight mutant-specific PCR primers (Table 1) were designed using the program Primer 3 (Rozen and Skaletsky 2000). These CYC2GENE primers (Table 1) are capable of amplifying sequence from the closely related group Vicia (S. Sveinsson, personal 
communication) and might even be useful in other related legume genera (e.g. for phylogenetics). Primer tests using the wild-type-specific primers were conducted using DNA from the parental varieties. Differential amplification between wild-type and $h d d$ varieties occurred in a number of the primer pairs. Using one such set of primers, CYC2GENE4/9 (sequence details and the position in the ORF of all primers are given in Table 1), DNA samples from the F2 population were analyzed. The CYC2GENE4/9 primer pair amplifies a DNA fragment approximately 100bp in length in wild-type Lathyrus odoratus but does not amplify in any plants with a hdd phenotype. This is expected as the CYC2GENE4 primer binds to the distal part of the TCP-box, which has been disrupted in the mutant allele of $L o C Y C 2$. Mutant-specific primers were then used to verify the results. This was done to ensure that the lack of PCR amplification seen when using wild-type primers was caused by a change in DNA sequence that influenced the primer binding site. Two sets of primers, LoHoodedGene4L/4R and LoHoodedGene3L/2R, were used to amplify sequence around bp 417 of $L o C Y C 2$ to ensure that the change in sequence that was observed during genome walking was correct.

SEM

All SEM data were collected using a Hitachi S-2600N scanning electron microscope at an acceleration voltage of $20 \mathrm{kV}$. Petals were extracted from blooming flowers and mounted onto scanning electron microscope stubs using double-sided adhesive tape. Fresh tissue was used under low-vacuum conditions (20 Pa) due to the ready availability of flowers and time constraints. The petal surface was examined to provide visible differences in cell type that can be used as micromorphological markers to determine petal identity. The adaxial surface was examined as this surface (the one displayed to pollinators) has the greatest difference as determined by previous studies (Cronk 2006; Feng et al. 2006; Ojeda et al. 2009; Ojeda et al. 2012). Seven different 
cultivated varieties of Lathyrus odoratus were examined to verify that any trends in cell shape that were observed were conserved traits: three $h d d$ varieties (Annie B Gilroy, Lady's Bonnet and Lady Grisel Hamilton) and four wild type varieties (Cupani, Miss Wilmott, North American Beautifier Scarlet, Painted Lady). One to five samples of each flower petal type were used to generate the results.

\section{Results}

Using standard primers LoCYC2 transcript is detected in the standard petals of wild type Lathyrus odoratus but not in the hooded mutant

Reverse Transcriptase PCR (RT-PCR) reactions were carried out using Lathyrus odoratus floral cDNA and primers designed to amplify CYCLOIDEA homologues (CYC1 and CYC2) in the closely related species Pisum sativum. Sequencing showed that these primers amplified almost identical genes in L. odoratus (GenBank MG281954 and MG281955).

Using these primers, we found $\mathrm{L} o \mathrm{CYCl}$ to be strongly expressed in the standard petals of both $h d d$ and non- $h d d$ varieties. In addition to being expressed in the standard petal, LoCYCl is also weakly expressed in wing petals (Figure 2).

LoCYC2 is strongly expressed in the standard petal of wild-type L. odoratus, but not at all in wing and keel petals (Figure 2). In $h d d$ plants, $L o C Y C 2$ transcript was not amplified in any petal type. Identical results were found for all hooded varieties tested (Figure 2). This suggests that either the $h d d$ mutation has resulted a change of expression pattern in this gene or that the sequences complementary to the primer sequence are not present in the mutant. 
A major change in LoCYC2 sequence is found in hooded Lathyrus odoratus

To determine the cause of the differential amplification patterns that were observed in the RT-PCR analysis, gene sequence from wild-type and hdd Lathyrus odoratus was examined. The primers used for $C Y C 2$ RT-PCR amplified the genomic DNA of wild type plants but did not amplify the genomic DNA of the $h d d$ mutant, indicating that a change of gene sequence within the ORF has occurred in the hdd mutant that affects at least one of the primer binding sites. The full-length sequences of both the wild type and mutant genes were therefore determined using genome walking techniques.

CYC2 sequence from wild-type L. odoratus showed very high sequence similarity (95\%) to the sequence found in the closely related species Pisum sativum (Wang et al. 2008). The few differences in protein sequence between Pisum and Lathyrus appear to be caused by homopolymer repeats with different amino acid repeat numbers. The TCP-box of LoCYC2 occurs at base pairs (bp) 380-521 and the R-box at bp 758-803 (Figure 3). The open reading frame of LoCYC2 is 1274 bp in length.

However, the sequence of the $h d d$ mutant allele shows some very large differences when compared to wild-type $\operatorname{LoCYC} 2$ sequence. The first portion of the $5^{\prime}$ sequence is identical to that found in the wild-type, but the sequences diverge at bp 417 (Figures 3 and 4), i.e. halfway through the conserved TCP-box and before the conserved R-box. Two sets of primers, LoHoodedGene4L/4R and LoHoodedGene3L/2R, were used to amplify sequence around bp 417 of $L o C Y C 2$ to ensure that the change in sequence that was observed during genome walking was not a sequencing artifact.

A large number of CYC2GENE primer pairs (Table 1) were designed specifically to amplify different portions of the L. odoratus homolog of CYC2. These consistently amplified the 
wild type gene but predictably failed to amplify the $h d d$ mutant. The change in DNA sequence observed in $h d d$ L. odoratus may be caused by an insertion or deletion (indel) event that disrupts the gene. In an attempt to sequence past a putative insertion, over 900 nucleotides were sequenced following the change. However, the $3^{\prime}$ portion of the $L o C Y C 2$ gene, including the functionally relevant $\mathrm{R}$ domain, was not located suggesting that either the insertion is very large or the $3^{\prime}$ region of the gene has been entirely deleted.

We therefore conclude that all hooded plants carry a mutant allele in which the last two thirds of the gene are replaced by at least $900 \mathrm{bp}$ of unrelated sequence as the result of an indel event. The altered sequence of the mutant encodes a truncated protein that lacks all or part of both the highly conserved domains and is likely non-functional. The protein is identical to WT for the first 139 amino acids but then is completely different, lacking the remainder of the WT amino acid sequence (of 460 aa). The mutant sequence has numerous stop codons (the first one after only a further 63 nucleotides) (Figures 3 and 4). BLAST searches of the replacement sequence fail to find any similar sequences in the databases.

It is therefore clear that the cause of the failure of the RT-PCR primers to detect transcript in hooded varieties is that the part of the gene amplified (see Methods) is simply missing in these varieties. It is possible that the truncated part of the gene is expressed, but if so this is unlikely to be functional as it is missing all or part of both highly conserved domains.

\section{The mutant allele of LoCYC2 segregates with the hooded phenotype}

The results of the sequence analysis show that there is an association between the $h d d$ phenotype and a major change in sequence in the LoCYC2 gene. An F2 co-segregation analysis was used to expand the sample size, and determine if the $h d d$ phenotype was consistently 
inherited with the mutant allele of LoCYC2. The hooded ( $h d d)$ gene has long been known to be recessive (Punnett 1919). F1 plants were generated from homozygous $h d d$ and wild type parent and an F2 population of 118 plants was examined using primers CYCGENE4/9. Of these, 25 possessed the hdd phenotype and 93 the wild type (expected: 29.5 and 88.5).

The CYC2GENE4/9 set of primers amplifies a DNA fragment 105bp in length in wildtype Lathyrus odoratus (positions 496-600; Table 1) but does not amplify in plants with a hdd phenotype. This is expected as the CYC2GENE4 primer binds to the later portion of the TCPbox domain which has been disrupted in the mutant allele of LoCYC2. Mutant-specific primers were then used to verify the results. This was done to confirm that the lack of PCR amplification seen when using wild-type primers was caused by a change in DNA sequence that influenced the primer binding site.

All hdd plants were homozygous for the $C Y C 2$ mutant allele. All wild type plants were either heterozygous, or homozygous for the wild type allele. Co-segregation was therefore perfect for this sample.

Epidermal cell type differs between hooded and wild-type Lathyrus odoratus

Scanning Electron Microscopy (SEM) was used to examine cell types present on the adaxial and abaxial surfaces of standard, wing and keel petals in Lathyrus odoratus. Although not all papilionoid legumes in the IRLC clade have discernable differences in petal-specific cell types (Ojeda et al. 2009), cell shape and size can be used as a tool to determine petal dorsiventral identity in L. odoratus. Cell shape and cell type were examined in all petals of the $h d d$ floral mutant of $L$. odoratus. The standard petal of $h d d$ plants has taken on cell characteristics found in wing petals of wild-type flowers (Figure 5), suggesting that the mutation is homeotic in nature. 
Homeosis is the process by which the characteristics of one part of an organism are transferred to another, usually by heterotopic gene expression patterns. In this case, characteristics of lateral petals have been transferred to the dorsal petal (Cronk 2006; cf. Citerne et al. 2006).

The adaxial surface of the standard petal of wild-type L. odoratus is composed primarily of elongate tabular rugose (TRS) cells. The adaxial surface of wild-type wing petals is composed primarily of papillose knobby rugose (PKR) cells. The difference in appearance between the cells of the standard petal and those found in the wing petal is easily distinguishable, for the cells found in the wing petals are nearly isodiametric and have a surface that is much more lens-like, which is a characteristic of PKR cells. The adaxial surface of keel petals of wild-type L. odoratus is composed of brick-shaped, tabular flat (TFS) cells.

The adaxial surface of $h d d$ standard petals is comprised of PKR cells that are indistinguishable from those found in the wing petals of both $h d d$ and wild-type L. odoratus petals (Figure 5). The adaxial surface of $h d d$ wing and keel petals is unchanged in comparison to wild-type petals.

\section{Discussion}

A mutation in LoCYC2 causes the hooded phenotype

The hooded phenotype of Lathyrus odoratus is constantly associated with a massive sequence truncation of $\mathrm{LoCYC} 2$ at nucleotide 417 . Thus a mutation in the CYC2 gene appears to be the basis of the hooded (hdd) mutant. The L. odoratus hdd mutation is therefore directly equivalent to other $C Y C 2$ loss of function mutations known in other legume species. In Lotus, the 
squared standard (squ1) mutant is caused by a point mutation of CYC2 near the 3 ' end of a very short intron that causes the protein to be incorrectly spliced (Feng et al. 2006). It has a phenotype that affects the standard petal. In pea (Pisum sativum) the lobed standard mutant (lst) is also caused by a change in $C Y C 2$, which likewise affects the morphology of the standard petal. In contrast, we detected no sequence or expression differences between the $h d d$ mutant and the wild type in $L o C Y C 1$.

\section{LoCYC2 plays a role in dorsal petal identity}

The hooded mutant is never bicoloured. Bateson (1913) suspected that there could be close linkage between $h d d$ and the gene that controls the bicolour trait. However, in the time since this prediction was made even the most closely linked genes might be expected to have undergone a recombination event. An alternative explanation is that they are the same gene. If the mutation is homeotic in nature, then the abolition of dorsal petal identity characteristic of the standard petal (for which CYC2 is required) might cause the standard petal to be much more similar to a wing and therefore a difference in colour between standard and wing would be impossible. The observable petal phenotypes support this interpretation. The hdd mutants in our F2 population are all unicolourous with the standard petal taking on the colour expected in the wings. As mentioned above, the process of one part of an organism coming to resemble another part is termed homeosis and has previously been demonstrated in legume flowers (Citerne et al. 2006). This is ironic as it was Bateson himself who coined the term homeosis (Bateson, 1894), although in this instance he apparently failed to recognize it.

To further investigate the homeotic hypothesis, we examined another marker of standard petal identity: epidermal cell morphology. The patterns of petal micromorphology are also 
consistent with homeosis and show that the standard petal of $h d d$ flowers has taken on wing-like characters.

\section{Conclusion}

We conclude that $L o C Y C 2$ is an adaxial petal identity gene capable of leading to homeotic transformation of the standard petal to wing-like morphology in loss of function mutants. This is consistent with work on Cadia, a papilionoid legume with five identical dorsalized petals (Citerne et al. 2006), a case of CYC2 gain of function causing homeosis. While CYC2 homologs are expressed only in the standard petals in typical papilionoid legumes such as Lotus and Pisum, the gene (referred to as LegCYC1B in Citerne et al.'s 2006 paper) is strongly expressed in every petal of Cadia. The actinomorphic flowers observed in Cadia can be compared to the overexpressed Lotus japonicus LjCYC2 line SH0578 that displays LoCYC2 expression in every petal and has a completely dorsalized phenotype (Feng et al. 2006). The over-expression of legume $C Y C 2$ leads to expansion of dorsal petal identity, whereas loss of function mutations in the gene lead to flowers where dorsal identity is incomplete or has been lost (Citerne et al. 2006; Feng et al. 2006; Wang et al. 2008). Our work on the Lathyrus odoratus hooded mutant therefore further confirms previous findings that genes in the legume CYCLOIDEA2 clade ( $\operatorname{LegCYC2}$ ) act as dorsal petal identity genes that are vital for standard petal development and floral bilateral symmetry in the Leguminosae.

\section{Acknowledgements}


We would like to thank David Kaplan, and Seane Trehearne for their help in the growth and maintenance of Lathyrus odoratus populations. Farnaz Pournia and Evelyn Carpenter helped collect tissue samples and maintain L. odoratus plants during the F2 trial. We would like to acknowledge Dorothy Cheung, Nyssa Temmel, Isidro Ojeda, Julia Nowak, Ji Yong Yang, Armando Geraldes, and Sæmundur Sveinsson for their help over the course of the project. We gratefully acknowledge the support of an NSERC discovery grant to Quentin Cronk.

\section{References}

Bateson, W. 1894. Materials for the Study of Variation treated with especial regard to discontinuity in the origin of species. Macmillan, New York.

Bateson, W. 1902. Mendel's Principles of Heredity - A defence. Cambridge University Press, Cambridge.

Bateson, W. 1905. Notes on the progress of Mendelian studies. Reports to the Evolution Committee of the Royal Society of London, 2: 119-131.

Bateson, W. 1913. Mendel's Principles of Heredity. Cambridge University Press, Cambridge.

Bateson, W., Saunders, E.R., and Punnett, R.C. 1906. Experimental studies in the physiology of heredity. Reports to the Evolution Committee, Royal Society of London, 3: 2-11. 
Broholm, S.K., Tätiharju, S., Laitinen, R.A.E., Albert, V.A., Teeri, T.H., and Elomaa, P. 2008. A TCP domain transcription factor controls flower type specification along the radial axis of the Gerbera (Asteraceae) inflorescence. Proc. Natl. Acad. Sci. U.S.A. 105(26): 91179122.

Carlson, E.A. 2004. Mendel's legacy, the origin of classical genetics. Cold Spring Harbor Laboratory Press, Cold Spring Harbour, New York.

Citerne, H.L., Luo, D., Pennington, R.T., Coen, E., and Cronk, Q.C.B. 2003. A phylogenomic investigation of CYCLOIDEA-like TCP genes in the Leguminosae. Plant Physiol. 131: 1042-1053.

Citerne, H.L., Pennington, R.T., and Cronk, Q.C.B. 2006. An apparent reversal in floral symmetry in the legume Cadia is a homeotic transformation. Proc. Natl. Acad. Sci. U.S.A. 103(32): 12017-12020.

Cronk, Q.C.B. 2006. Legume flowers bear fruit. Proc. Natl. Acad. Sci. U.S.A. 103(13): 48014802.

Cubas, P. 2002. Role of TCP genes in the evolution of key morphological characters in angiosperms. In Developmental Genetics and Plant Evolution. Edited by Q.C.B. Cronk, R.M. Bateman and J.A. Hawkins. Taylor and Francis, London, pp. 247-266, 
Cubas, P., Lauter, N., Doebley, J., and Coen, E. 1999. The TCP domain: a motif found in proteins regulating plant growth and development. Plant J. 18(2): 215-222.

Fambrini, M., and Pugliesi, C. 2017. CYCLOIDEA 2 clade genes: key players in the control of floral symmetry, inflorescence architecture, and reproductive organ development. Plant Molecular Biology Reporter, 35: 20-36.

Feng, X., Zhao, Z., Tian, Z., Xu, S., Luo, Y., Cai, Z., Wang, Y., Yang, J., Wang, Z., Weng, L. and Chen, J. 2006. Control of petal shape and floral zygomorphy in Lotus japonicus. Proc. Natl. Acad. Sci. U.S.A. 103: 4970-4975.

Hileman L.C. 2014a. Bilateral flower symmetry - how, when and why? Curr. Opin. Plant Biol. 17: $146-152$.

Hileman L.C. 2014b. Trends in flower symmetry evolution revealed through phylogenetic and developmental genetic advances. Philos. Trans. R. Soc. Lond. B Biol. Sci. 369: art.20130348.

Hileman, L.C., Kramer, E.M., and Baum, D.A. 2003. Differential regulation of symmetry genes and the evolution of floral morphologies. Proc. Natl. Acad. Sci. U.S.A. 100(22): 12814 12819. 
Luo, D., Carpenter, R., Vincent, C., Copsey, L., and Coen, E. (1996). Origin of floral asymmetry in Antirrhinum majus. Nature, 383: 794-799.

Martín-Trillo, M., and Cubas, P. 2009. TCP genes: a family snapshot ten years later. Trends Plant Sci. 15(1): 31-39.

Ojeda, I., Francisco-Ortega, J., and Cronk, Q.C.B. 2009. Evolution of petal epidermal micromorphology in Leguminosae and its use as a marker of petal identity. Ann. Bot. 104(6): 1099-1110.

Ojeda, I., Santos-Guerra, A., Caujapé-Castells, J., Jaén-Molina, R., Marrero, A., and Cronk, Q.C.B. 2012. Comparative micromorphology of petals in Macaronesian Lotus (Leguminosae) reveals a loss of papillose conical cells during the evolution of bird pollination. Int. J. Plant Sci. 173(4): 365-374.

Ojeda, D. I., Jaén-Molina, R., Santos-Guerra, A., Caujape-Castells, J., and Cronk, Q.C.B. 2017. Temporal, but not spatial, changes in expression patterns of petal identity genes are associated with loss of papillate conical cells and the shift to bird pollination in Macaronesian Lotus (Leguminosae). Plant Biology, 19(3): 420-427.

Punnett, R.C. 1919. Mendelism, 5 edn. MacMillan, London.

Punnett, R.C. 1923. Lathyrus odoratus. Bibliographica Genetica, 1: 69-82. 
Rice, G. 2002. The Sweet Pea Book. Timber Press, Portland, OR.

Rozen, S., and Skaletsky, H.J. 2000. Primer3 on the WWW for general users and for biologist programmers. In Bioinformatics Methods and Protocols: Methods in Molecular Biology. Edited by S. Krawetz and S. Misener, S. Humana Press, Totowa, NJ. pp. 365-386.

Senn, H.A. 1938. Experimental data for a revision of the genus Lathyrus. Am. J. Bot. 25(2): 6778.

Wang, Z., Li, X., Luo, Y., Wang, L., Xu, S., Yang, J., Weng, L., Sato, S., Tabata, S., Ambrose, M., and Rameau, C. 2008. Genetic control of floral zygomorphy in the pea (Pisum sativum L.). Proc. Natl. Acad. Sci. U.S.A. 105(30): 10414-10419.

Westerkamp, C. 1993. The co-operation between the asymmetric flower of Lathyrus latifolius (Fabaceae-Vicieae) and its visitors. Phyton (Horn, Austria), 33: 121-137.

Xu, S., Luo, Y., Cai, Z., Cao, X., Hu, X., Yang, J., and Luo, D. 2013. Functional diversity of CYCLOIDEA-like TCP genes in the control of zygomorphic flower development in Lotus japonicus. Journal of Integrative Plant Biology, 55(3): 221-231.

Xu, Z., Cheng, K., Li, X., Yang, J., Xu, S., Cao, X., Hu, X., Xie, W., Yuan, L., Ambrose, M. and Chen, G. 2016. Transcriptional and post-transcriptional modulation of SQU and KEW 
activities in the control of dorsal-ventral asymmetric flower development in Lotus japonicus. Molecular Plant, 9(5): 722-736.

Zhong, J., and Kellogg, E.A. 2015. Duplication and expression of CYC2 $\square$ like genes in the origin and maintenance of corolla zygomorphy in Lamiales. New Phytologist, 205(2): 852-868. 
Table 1. Primer sequences used to amplify overlapping parts of the full length CYC2 gene, both wild type (WT) and the hooded mutant (hdd). $\mathrm{F}=$ forward and $\mathrm{R}=$ reverse. The starting position of the primer in the ORF (or mutant sequence).

\begin{tabular}{|l|l|l|l|}
\hline Primer Name & Primer Sequence & $\begin{array}{l}\text { Primer } \\
\text { Target }\end{array}$ & $\begin{array}{l}\text { Primer start } \\
\text { site in oRF (nt) }\end{array}$ \\
\hline CYC2GENE1 (F) & AATAACATTGCGGTTCCGATGCCG & WT & 280 \\
\hline CYC2GENE2 (R) & GCCAATCAAGTGTGTTGCTGGCTT & WT & 517 \\
\hline CYC2GENE3 (R) & TCTTTCCCTTGCTCTTGCTCTTGC & WT & 798 \\
\hline CYC2GENE4 (F) & GCCAGCAACACACTTGATTGGCTT & WT & 496 \\
\hline CYC2GENE5 (R) & CGTGGATTGGTGCATTCTTCCCAT & WT & 1256 \\
\hline CYC2GENE6 (F) & ATGGGAAGAATGCACCAATCCACG & WT & 1233 \\
\hline CYC2GENE7 (F) & AGCAAGAGCAAGAGCAAGGGAAAG & WT & 774 \\
\hline CYC2GENE8 (R) & CGGCATCGGAACCGCAATGTTATT & WT & 303 \\
\hline CYC2GENE9 (R) & GGCATCGCCACCAACTTCACTATT & WT & 600 \\
\hline CYC2GENE10 (R) & AAGCCAATCAAGTGTGTTGCTGGC & WT & 519 \\
\hline CYC2GENE11 (F) & TCTTCAAGAGATGTTAGGGTTTGAC & WT & 468 \\
\hline CYC2GENE12 (R) & CCTCCTTTTGTAGGTTTAAGGAATC & WT & 676 \\
\hline CYC2GENE13 (F) & TCCCTTATAGTTCAAACCCTTATCC & WT & 5 \\
\hline CYC2GENE14 (R) & GAGAACTATGATGGTGAGTTGGAAT & WT & 181 \\
\hline CYC2GENE15 (F) & GCAATTAAGGATCTAACAAAAAGCA & WT & 538 \\
\hline CYC2GENE16 (R) & CTTGACTCCTTCATCTTACTTGCTC & WT & 767 \\
\hline CYC2GENE17 (R) & CTCTTGACTCCTTCATCTTACTTGC & WT & 769 \\
\hline CYC2GENE18 (F) & CAAGCAAAGAGACAACAATAGTGAA & WT & 561 \\
\hline LoHoodedGene1F & CCGAGTTGCTAACAGTTTTGTATTTAT & hdd & 995 \\
\hline LoHoodedGene1R & GGAGAGATATTATACGCATTGTTGAAT & hdd & 1186 \\
\hline LoHoodedGene2F & TAAAGCATATATTGACAACAACCACTC & hdd & 636 \\
\hline LoHoodedGene2R & GACTATATTGAATTTGGAAGTCAGAGG & hdd & 833 \\
\hline LoHoodedGene3F & GGTTCTCACTATGGAATTTCTTGTTT & hdd & 331 \\
\hline LoHoodedGene3R & TTTTAGAATTGGTTAGCCATTAATTTG & hdd & 482 \\
\hline LoHoodedGene4F & TCACTATGGAATTTCTTGTTTCCTTAC & hdd & 336 \\
\hline LoHoodedGene4R & AAAAATTTAATGACAATTTCGGATGT & hdd & 541 \\
\hline
\end{tabular}




\section{Figure captions}

Figure 1: Lathyrus odoratus flowers, showing the difference between (A) wild-type ('Cupani') and (B) the hooded form ('Annie B Gilroy'). Petal types (dorsal: standard; lateral: wings and ventral: keel) are indicated with arrows.

Figure 2: Expression patterns of genes in individual petals of hooded (Annie B. Gilroy, Lady's Bonnet, Miss Ellen Wilmott) and wild-type (Cupani, America) Lathyrus odoratus. Standard, wing and keel petals have been abbreviated to S, W, and K respectively. Lathyrus homologs of CYC1 and CYC2 were examined and Histone4 (H4) was used as a positive control.

Figure 3: Gene diagrams for the wild-type (above) and hooded (below) alleles of CYC2. Regions encoding important protein functional domains such as the TCP-box and the R-box are also labelled.

Figure 4: The aligned protein sequences of Lathyrus odoratus CYC2 homologs for wild-type and the hooded mutant allele. The boxes indicate conserved sequence. The hooded allele is truncated, with unalignable sequence followed by a premature stop codon. 
Figure 5: Adaxial epidermal cell types present in standard (left) and wings (right) of wild-type (A,B) and hooded (C,D) Lathyrus odoratus. In the hooded floral mutant of L. odoratus, the epidermis of the standard petal has taken on wing epidermis characteristics. This pattern was seen for all hooded mutants examined ('Annie B Gilroy' is illustrated). The white scale bars represent $100 \mu \mathrm{m}$. 


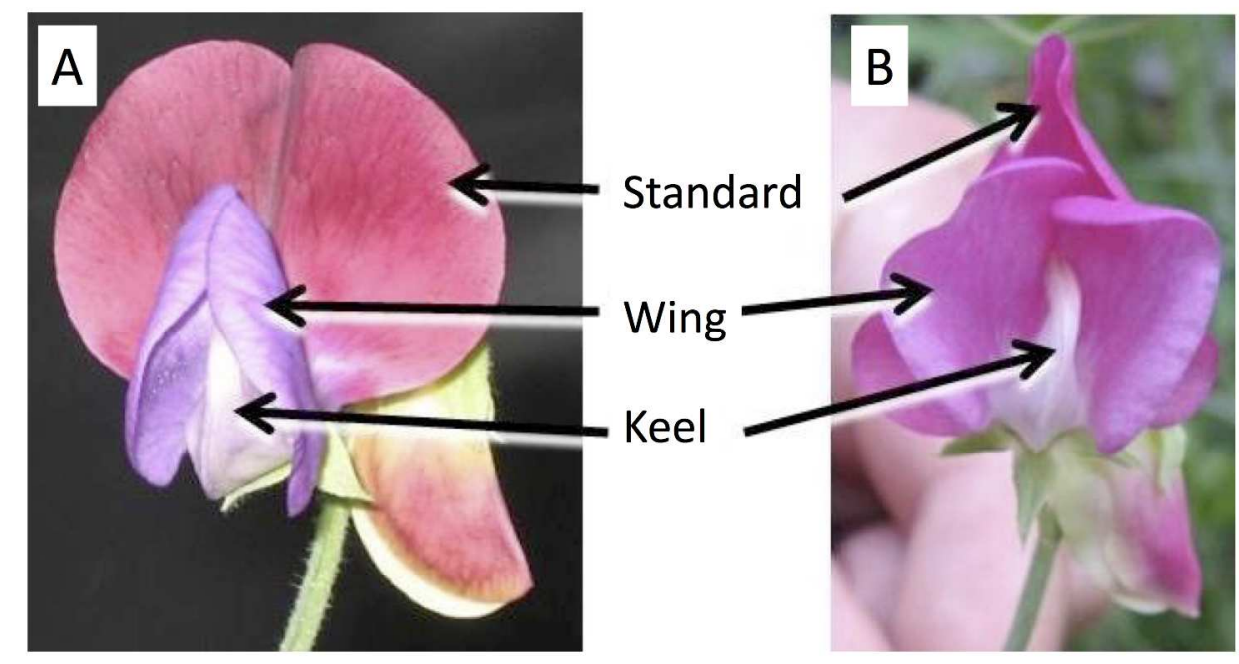

Figure 1: Lathyrus odoratus flowers, showing the difference between (A) wild-type ('Cupani') and (B) the hooded form ('Annie B Gilroy'). Petal types (dorsal: standard; lateral: wings and ventral: keel) are indicated with arrows.

$275 \times 154 \mathrm{~mm}(300 \times 300 \mathrm{DPI})$ 


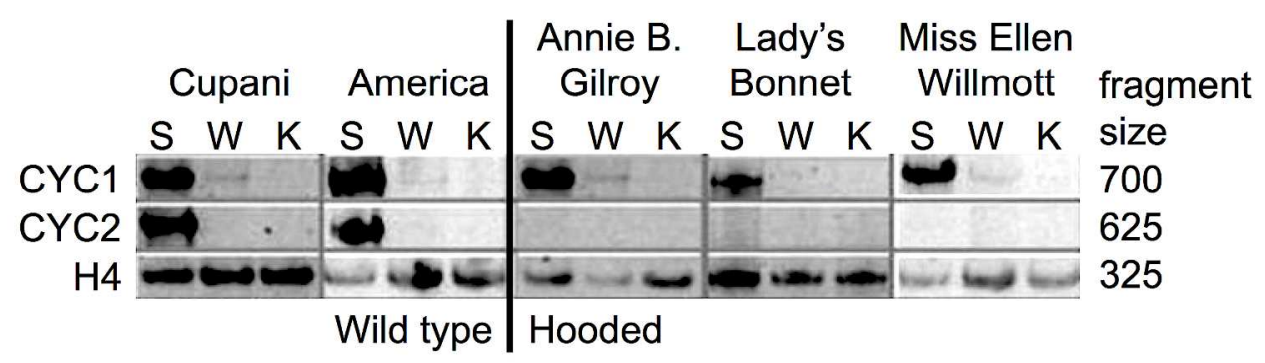

Figure 2: Expression patterns of genes in individual petals of hooded (Annie B. Gilroy, Lady's Bonnet, Miss Ellen Wilmott) and wild-type (Cupani, America) Lathyrus odoratus. Standard, wing and keel petals have been abbreviated to S, W, and K respectively. Lathyrus homologs of CYC1 and CYC2 were examined and Histone4 (H4) was used as a positive control.

$227 \times 77 \mathrm{~mm}(300 \times 300$ DPI $)$ 

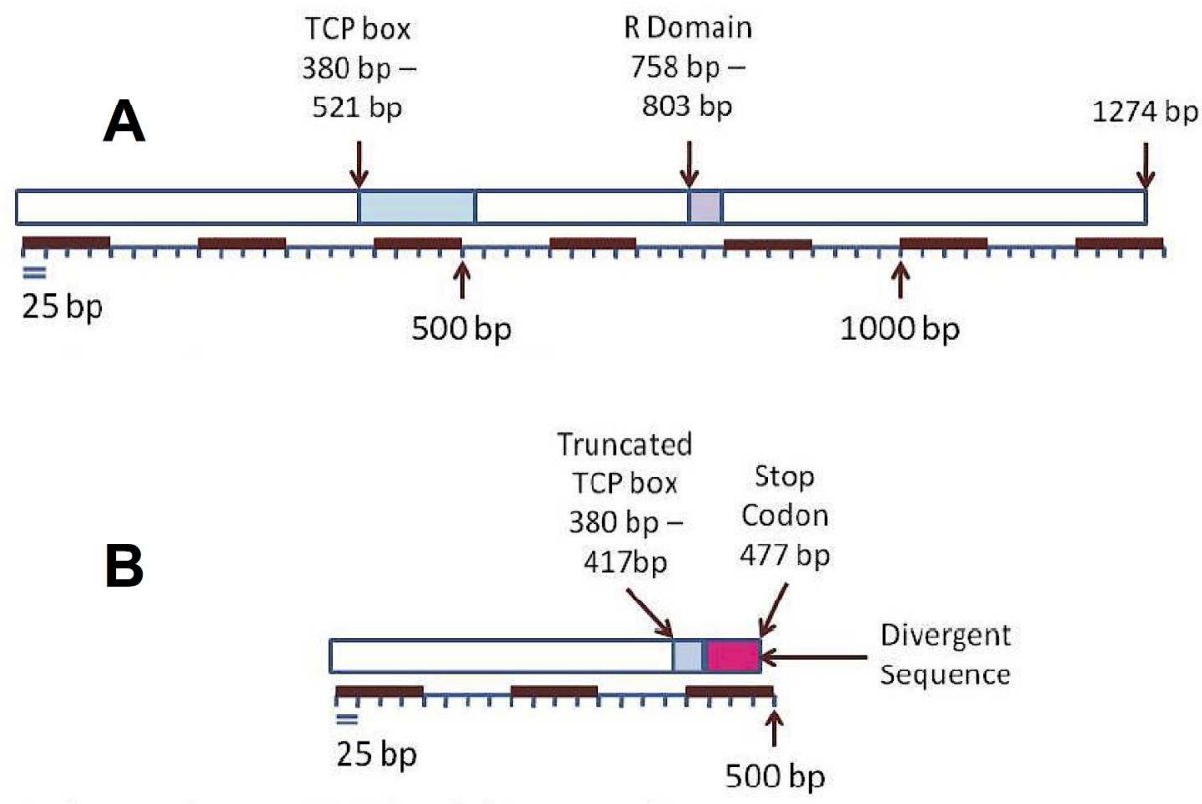

Figure 3: Gene diagrams for the wild-type (above) and hooded (below) alleles of CYC2. Regions encoding important protein functional domains such as the TCP-box and the R-box are also labelled.

$264 \times 175 \mathrm{~mm}(300 \times 300 \mathrm{DPI})$ 


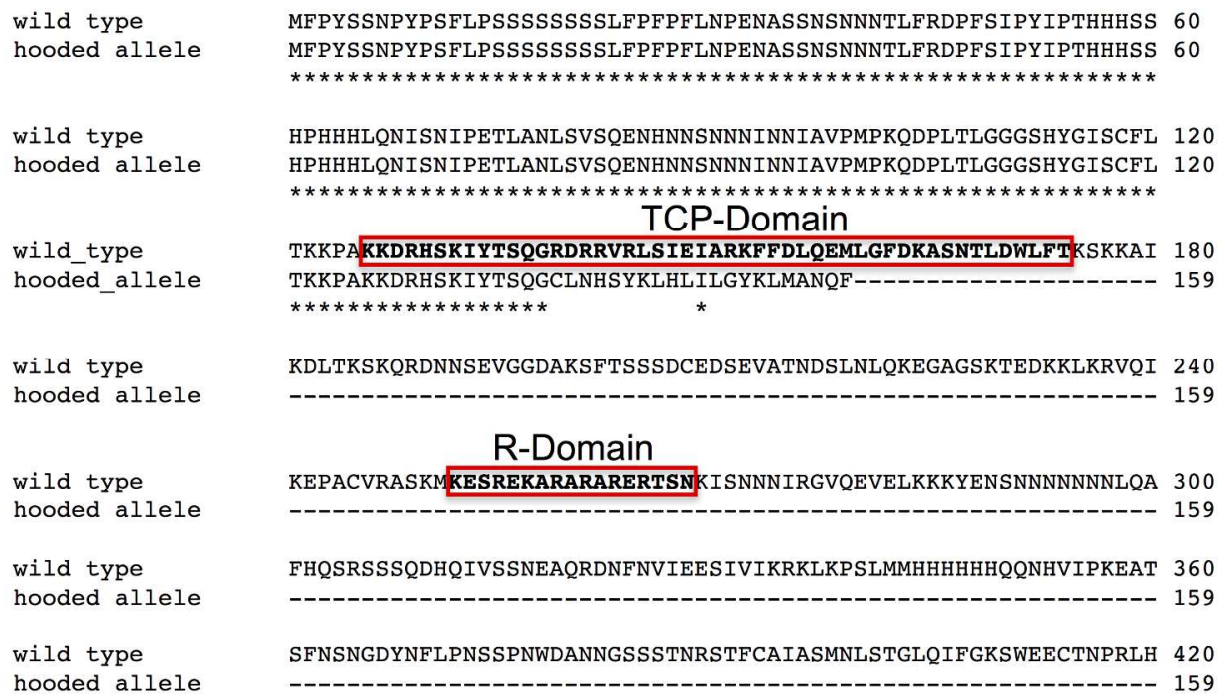

Figure 4: The aligned protein sequences of Lathyrus odoratus CYC2 homologs for wild-type and the hooded mutant allele. The boxes indicate conserved sequence. The hooded allele is truncated, with unalignable sequence followed by a premature stop codon.

$243 \times 146 \mathrm{~mm}(300 \times 300 \mathrm{DPI})$ 

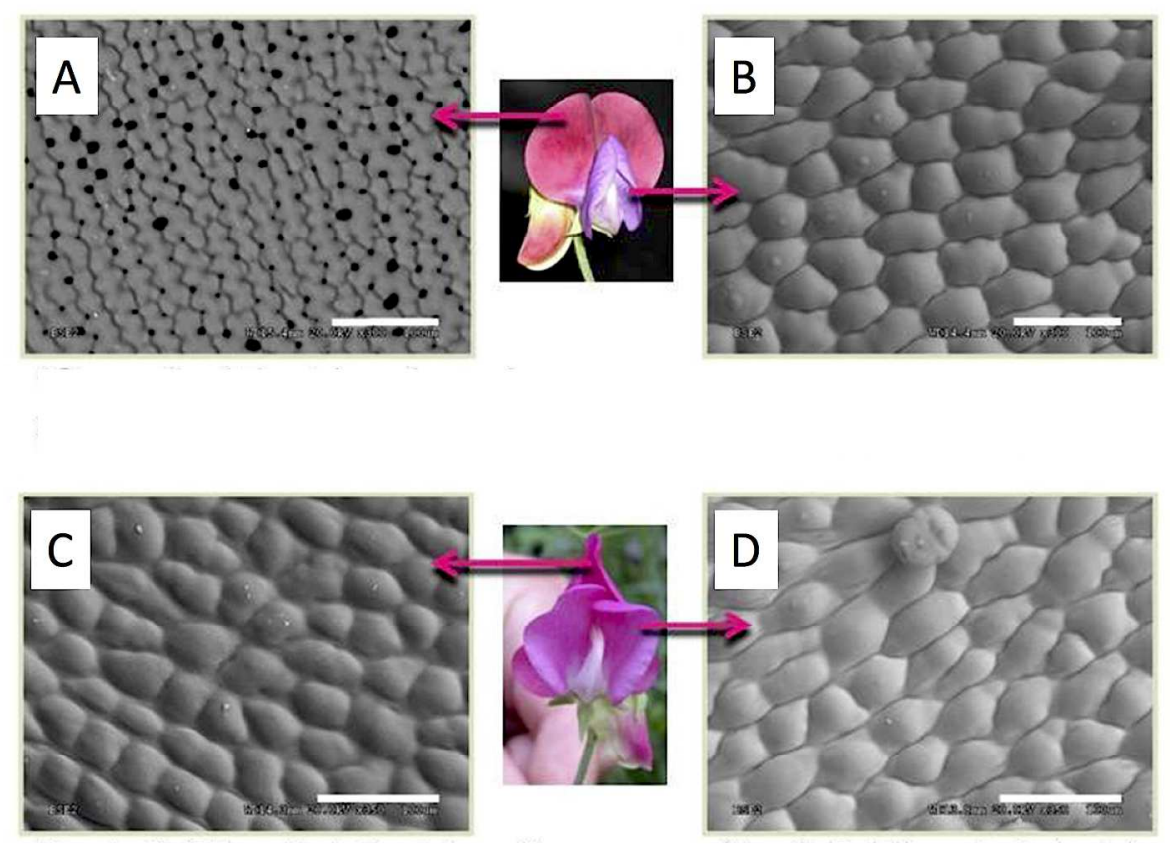

Figure 5: Adaxial epidermal cell types present in standard (left) and wings (right) of wild-type $(A, B)$ and hooded $(C, D)$ Lathyrus odoratus. In the hooded floral mutant of L. odoratus, the epidermis of the standard petal has taken on wing epidermis characteristics. This pattern was seen for all hooded mutants examined ('Annie B Gilroy' is illustrated). The white scale bars represent $100 \mu \mathrm{m}$.

$$
227 \times 157 \mathrm{~mm}(300 \times 300 \text { DPI) }
$$

